\title{
Sacrectomía parcial por abordaje posterior único
}

\author{
Pedro L. Bazán, Sergio Terraza, Álvaro E. Borri, Martín Medina \\ Hospital Interzonal General de Agudos “Gral. José de San Martín”, \\ La Plata, Buenos Aires
}

Recibido el 23-3-2016. Aceptado luego de la evaluación el 18-4-2017 • Dr. PEDRO L. BAZÁN • pedroluisbazan@ gmail.com

\begin{abstract}
RESUMEN
Introducción: Los tumores del sacro representan $<7 \%$ de los tumores espinales, prevalecen los tumores secundarios por mieloma múltiple o carcinomas de próstata, mama, pulmón o colón. El cordoma es el tumor maligno primario más frecuente y el tumor de células gigantes es la lesión benigna más común. Por su evolución, compromiso de estructuras extraóseas y la escasa respuesta a los tratamientos coadyuvantes, la cirugía es el tratamiento más utilizado, la vía de abordaje y la necesidad de instrumentación dependerán del tumor por tratar. Los objetivos de este trabajo son: evaluar el uso de la resección parcial del sacro, analizar la técnica quirúrgica y reconocer las complicaciones.

Materiales y Métodos: Cuatro pacientes con diagnóstico de tumor sacro ubicado por debajo de S1, que consultan por dolor y cuyas imágenes confirman la lesión. A todos se les realiza una resección en bloque por vía posterior preservando S1. Se describe la técnica.

Resultados: Los estudios anatomopatológicos revelaron: un cordoma, un tumor maligno de vaina nerviosa, un condrosarcoma y una metástasis de carcinoma prostático. Se preservó la función de S1 en todos los pacientes; uno tiene disfunción vesical permanente. Se observaron una dehiscencia de la herida, una infección y una fístula de líquido cefalorraquídeo. Todos permanecen sin la enfermedad tras un seguimiento de entre 6 y 24 meses.

Conclusiones: La resección parcial del sacro por abordaje posterior único se puede indicar cuando la lesión compromete desde S2 hacia distal y no hay compromiso sacroilíaco. La preservación de raíces es de vital importancia para garantizar mejores resultados posoperatorios y una menor tasa de infección.
\end{abstract}

Palabras clave: Tumor sacro; sacrectomía; cirugía espinal; abordaje quirúrgico; resección en bloque. Nivel de Evidencia: IV

\section{Partial sacrectomy by Single posterior approach}

\begin{abstract}
Introduction: Tumors of the sacrum represent less than $7 \%$ of spinal tumors. Secondary tumors due to multiple myeloma or prostate, breast, lung and colon carcinomas predominate. Chordoma is the most frequent primary malignant tumor and giant cell tumor is the most common benign lesion. For its evolution, involvement of extra bone structures and poor response to the adyuvant therapy, surgery is the most commonly used treatment, the approach and the need for instrumentation depend on tumor to treat. The objectives of this study are to evaluate the use of partial resection of sacrum, analyze the surgical technique and assess complications.

Methods: Four patients with diagnosis of sacral tumor below S1, who presented with pain and images confirming the injury. All underwent an en bloc resection by posterior via preserving S1. The technique is described.
\end{abstract}

Conflicto de intereses: Los autores no declaran conflictos de intereses. 
Results: The pathological results were: a chordoma, a malignant nerve sheath tumor, a chondrosarcoma and a metastasis of prostatic carcinoma. S1 function was preserved in all patients; one has permanent bladder dysfunction. Wound dehiscence, infection and cerebrospinal fluid fistula were detected. Patients are free of disease after a follow-up of 6-24 months. Conclusions: Partial resection of the sacrum using a unique posterior approach may be indicated when the injury involves from S2 distally and no sacroiliac involvement is observed. Root preservation is vital to guarantee the best postoperative results and a lower rate of infection.

Key words: Sacral tumor; sacrectomy; spinal surgery; surgical approach; en bloc resection. Level of Evidence: IV

\section{Introducción}

Los tumores del sacro son entidades de baja frecuencia, que abarcan del $1 \%$ al $7 \%$ de los tumores espinales. La mayoría son secundarios a diseminación de un mieloma múltiple o carcinomas de próstata, mama, pulmón o colon. El tumor maligno sacro más frecuente es el cordoma, y constituye más de la mitad, mientras que el tumor de células gigantes es la lesión benigna más frecuente. ${ }^{1}$ Estos tumores son invasivos y, debido a su gran agresividad, progresan rápidamente..$^{2-5}$

A causa de la baja respuesta terapéutica a la quimioterapia y la radioterapia, la cirugía es la primera modalidad en el manejo de este tipo de lesiones. Los avances en las técnicas quirúrgicas han permitido a los cirujanos efectuar resecciones en bloque con alta tasa de éxitos y baja morbimortalidad. Recientemente se han descrito nuevos abordajes quirúrgicos y técnicas para resecciones sacras con el objetivo de proteger mejor las raíces nerviosas y mejorar los resultados funcionales, preservando las estructuras circundantes y reduciendo el sangrado intraoperatorio sin comprometer el margen oncológico. ${ }^{6}$

El objetivo de este trabajo es evaluar el empleo de la resección parcial del sacro por vía posterior única, analizar la técnica quirúrgica y reconocer las complicaciones.

\section{Materiales y Métodos}

Se evaluaron cuatro pacientes, cuyo motivo de consulta general fue dolor lumbar bajo de tipo infiltrativo y disestesias en la región perineal, asociado a trastornos esfinte- rianos en el paciente 1 (Tabla). Se realizaron estudios por imágenes (radiografías, resonancia magnética y tomografía computarizada) y estudios de funcionalidad esfinteriana (paciente 1). En las imágenes de los pacientes 1 y 2 , se visualizó una lesión tumoral que comprometía desde S2 hasta $\mathrm{S} 4$, que correspondieron a un tumor maligno de la vaina nerviosa y a un cordoma, respectivamente (Figuras 1 y 2). En el paciente 3, se observó compromiso desde S3 hasta la región proximal de S4 (Figura 3), posteriormente, se diagnosticó una metástasis única de un carcinoma de próstata. El paciente 4 tenía compromiso de S4 hacia distal, que resultó ser un condrosarcoma.

Debido a las características imagenológicas, se planifica una resección parcial del sacro vía posterior por abordaje único en copa invertida, sin toma de biopsia prequirúrgica.

\section{Técnica quirúrgica \\ Resección parcial del sacro por abordaje posterior con incisión en copa invertida}

Antes de la inducción antibiótica con cefazolina, se coloca al paciente en decúbito ventral, con apoyos en tórax y crestas ilíacas. Se incide la piel, en forma de copa invertida (Figura 4), con el vértice a nivel de L5-S1, extendiéndose $5 \mathrm{~cm}$ por debajo y hacia fuera de las espinas ilíacas posterosuperiores. Luego, desde el vértice, se prolonga la incisión sobre la línea media hasta L4. Se realiza una disección cuidadosa subperióstica de la musculatura paravertebral, exponiendo a partir de la lámina y de los procesos transversos de L4 hasta la totalidad del sacro, retrayendo lateralmente el glúteo mayor. A continuación, se efectúa una laminectomía de L5 y S1 (S2

Tabla. Características demográficas de los pacientes

\begin{tabular}{|c|c|c|c|c|c|}
\hline Paciente & Sexo & Diagnóstico & Edad & Nivel de resección & Complicaciones \\
\hline 1 & F & Tumor de vaina nerviosa & 56 & S1-S2 & Dehiscencia de la herida \\
\hline 2 & M & Cordoma & 65 & S1-S2 & Fístula de líquido cefalorraquídeo \\
\hline 3 & M & Metástasis de cáncer de próstata & 49 & Proximal S2-S3 & Infección del sitio quirúrgico \\
\hline 4 & M & Condrosarcoma & 38 & Proximal S3 & Ninguna \\
\hline
\end{tabular}

$\mathrm{F}=$ femenino, $\mathrm{M}=$ masculino. 


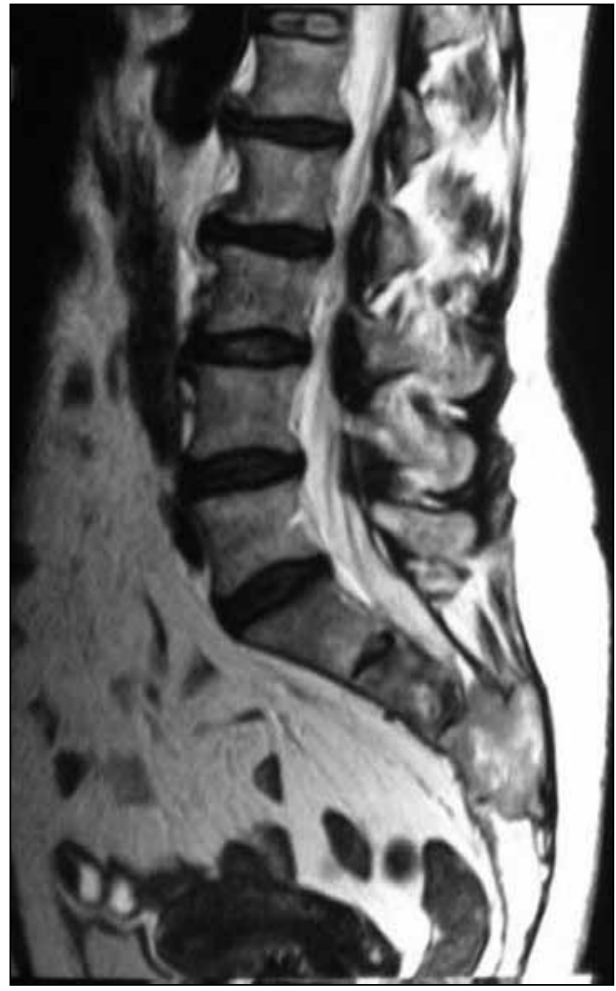

Figura 1. Mujer de 56 años, que consulta por lumbalgia baja y trastornos urinarios. Resonancia magnética, corte sagital paramediana T2, que muestra una lesión que compromete el conducto raquídeo desde S3 hasta S4, sin expansión de corticales. Diagnóstico anatomopatológico: tumor maligno de vaina nerviosa.

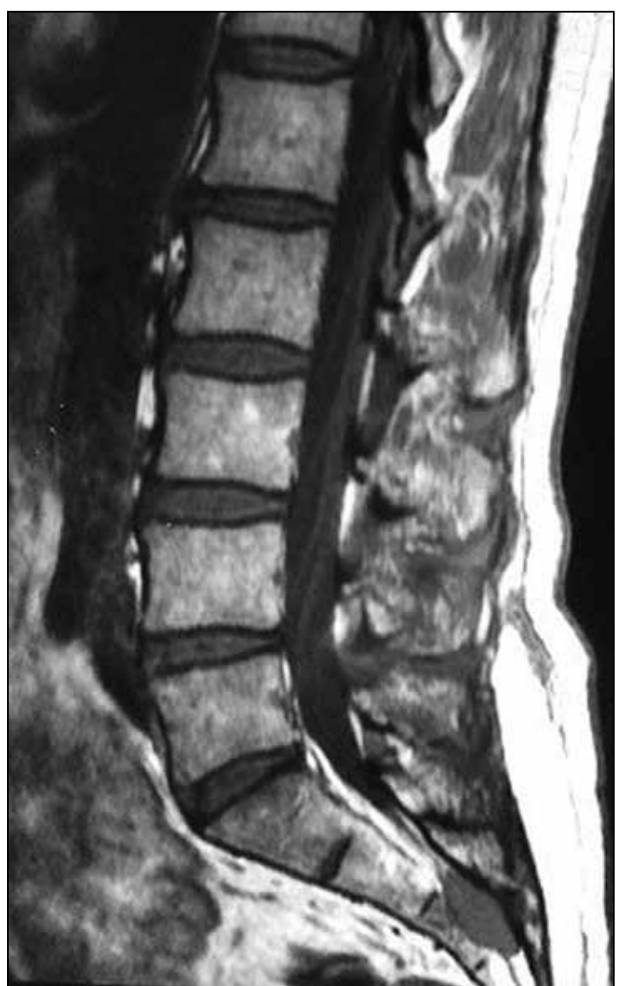

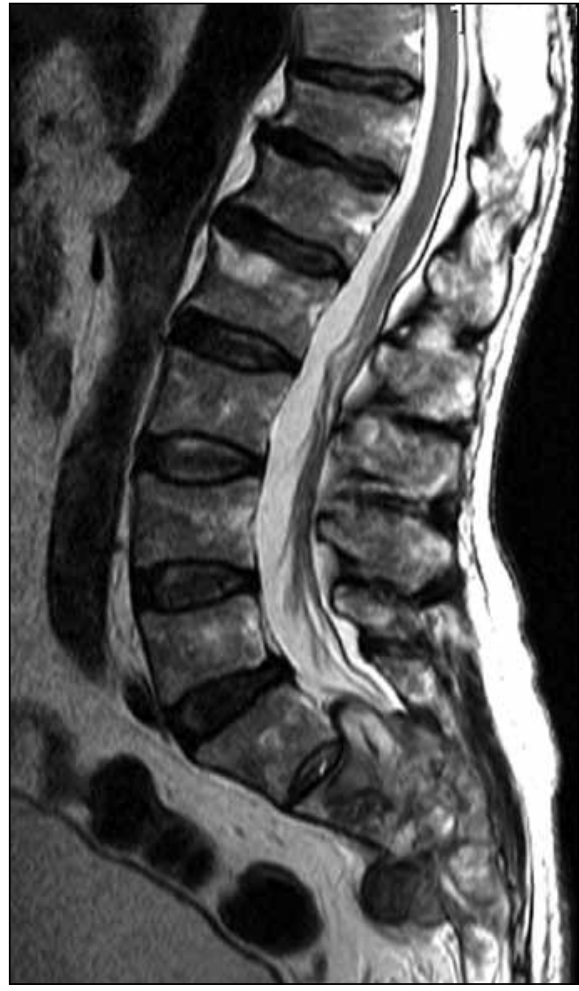

Figura 2. Hombre de 65 años, que consulta por lumbalgia baja y trastornos urinarios. Resonancia magnética, corte sagital paramediana $\mathrm{T} 2$, que muestra una lesión que compromete las estructuras óseas a distal de S1, con expansión de corticales. Diagnóstico anatomopatológico: cordoma.

Figura 3. Hombre de 49 años, que consulta por

lumbalgia baja. Resonancia magnética, corte sagital paramediana T1, que muestra una lesión intracanalar distal a S3, con expansión al cuerpo y al arco posterior. Diagnóstico anatomopatológico: metástasis única de carcinoma prostático.

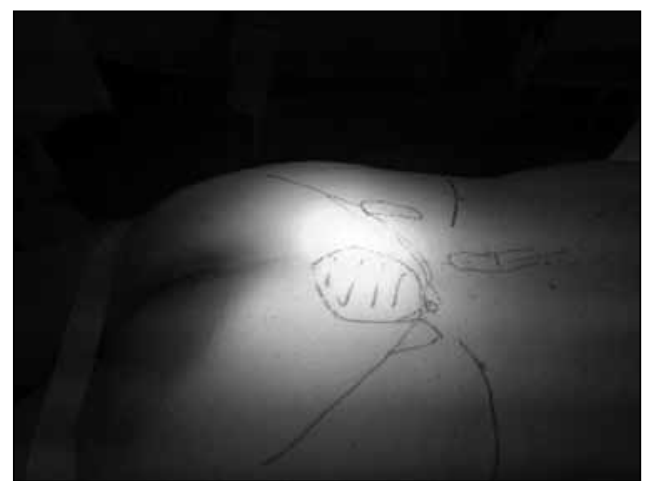

Figura 4. Colocación y planificación prequirúrgicas, con marcación en la piel de la localización del tumor sacro. 
según necesidad), intentando seguir la raíz de $\mathrm{S} 1$ hasta su salida foraminal y, según el compromiso de la raíz de S2 unilateral o bilateral, tratar de respetarla. Se liga y se secciona el saco dural. Luego, se seccionan los ligamentos sacrociático mayor y menor, y el músculo piramidal. Se procede a realizar una disección digital de todas las estructuras presacras, alcanzando a pasar de lado a lado una gasa vaselinada que ayuda para la disección presacra y como protección de las estructuras intrapelvianas al efectuar la osteotomía. Bajo visión directa de la raíz de S1 y con guía radioscópica, se lleva a cabo la osteotomía con escoplo ancho a partir del nivel superior seleccionado para la resección y luego la osteotomía por el borde lateral de S1 en dirección a la escotadura ciática mayor. La pieza se extrae abriendo lateralmente desde el lado que tenga menor compromiso tumoral disecando recto y estructuras vasculares de la fascia presacra. Las raíces que no estén comprometidas por el tumor se deben conservar. La pieza resecada se observa cuidadosamente para evaluar partes blandas y óseas (Figura 5). Se envía para estudio anatomopatológico. En estos casos, como no existe compromiso sacroilíaco, no se realiza reconstrucción del anillo pelviano. Se efectúa un correcto desbridamiento de tejidos desvitalizados y abundante lavado, en forma periódica, con solución fisiológica durante toda la cirugía. Se cierra la herida por planos, y se deja un drenaje por 48 horas como mínimo (Figura 6). La cirugía duró aproximadamente 5 horas. Los controles posoperatorios confirman la resección de la pieza (Figura 7).

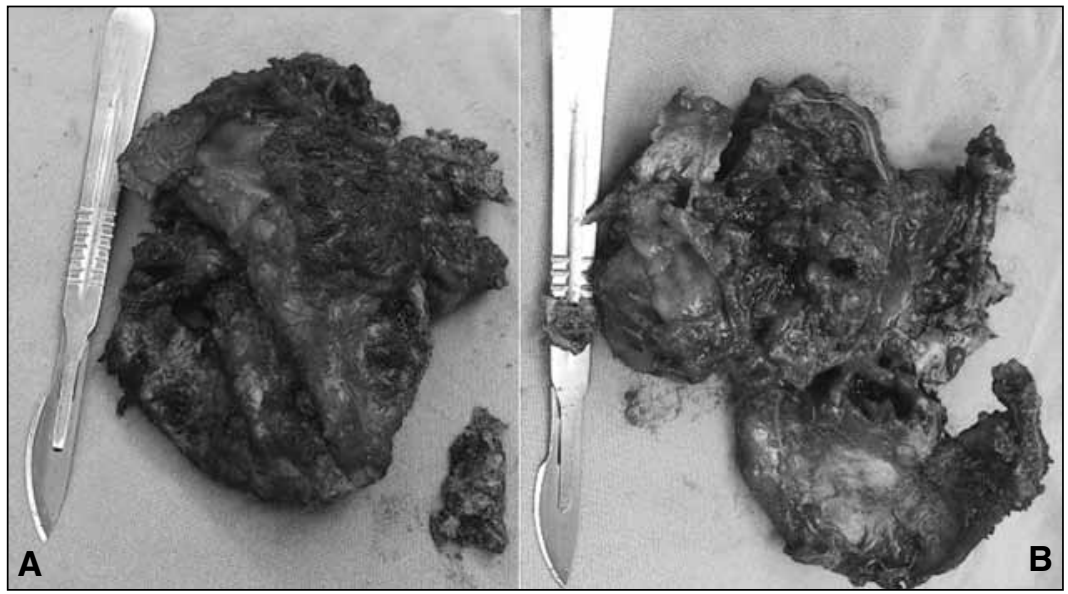

Figura 6. Imagen de una resección en bloque de un cordoma. Obsérvense la incisión en copa invertida con prolongación a cefálico, los dos tubos de hemosuctor y el catéter intradural.
Figura 5. Pieza quirúrgica de una resección en bloque del sacro, con diagnóstico anatomopatológico de cordoma. A. Cara posterior del sacro. B. Cara anterior del sacro.
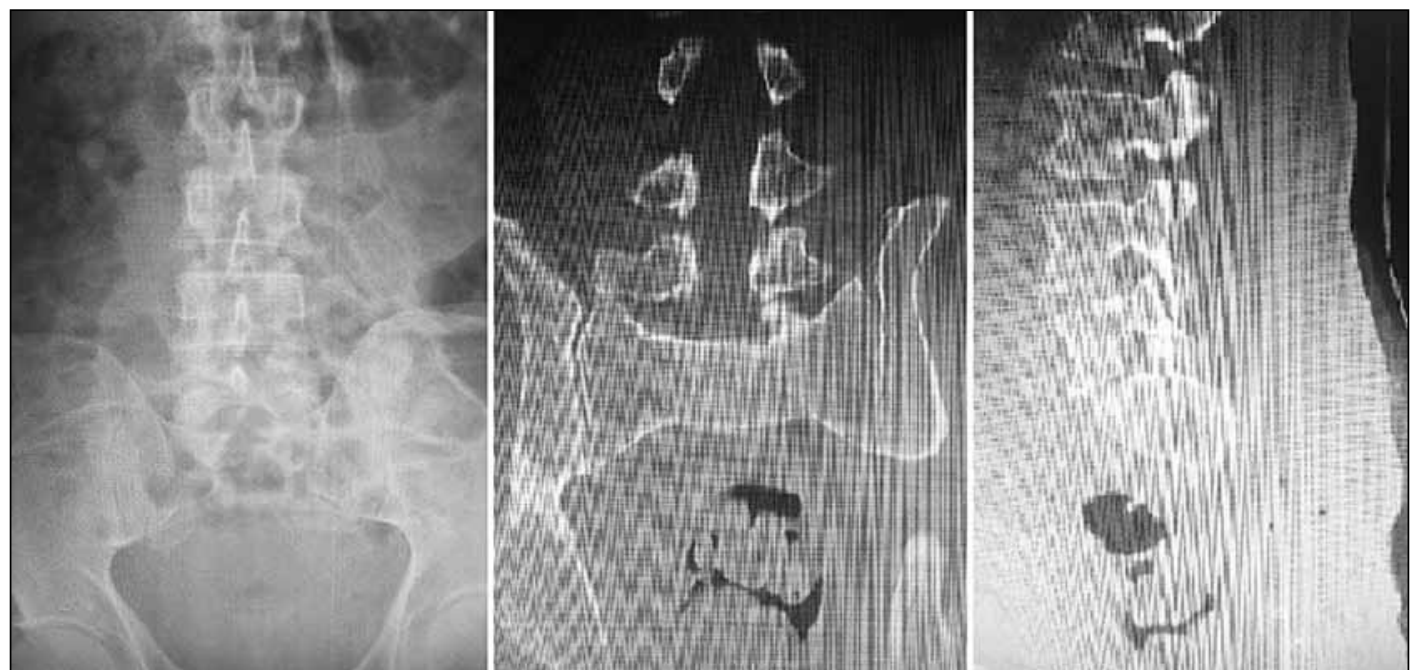

Figura 7. Estudios complementarios posoperatorios donde se observa la ausencia del extremo distal del sacro por la resección quirúrgica. 


\section{Resultados}

Se llevó a cabo la resección parcial del sacro por abordaje único por vía posterior en cuatro casos. La técnica descrita fue implementada por el mismo equipo quirúrgico. En ninguno de los pacientes, se tomó biopsia prequirúrgica. Los diagnósticos histopatológicos fueron: tumor maligno de la vaina nerviosa (paciente 1), cordoma (paciente 2), metástasis de carcinoma de próstata (paciente 3 ) y condrosarcoma (paciente 4). El síntoma común a todos fue el dolor. Sólo el paciente 1 tuvo síntomas de disfunción esfinteriana y el paciente 2 sufrió ciatalgia bilateral asociada a disestesias en el periné. Ninguno recibió quimioterapia ni radioterapia antes de la cirugía.

El nivel de resección fue S1-S2 en dos casos (pacientes 1 y 2), proximal a $S 2-S 3$ en un caso (paciente 3 ) y proximal a S3 en el restante (paciente 4). Se logró una resección amplia en bloque con margen de tejido sano en los cuatro pacientes.

Las complicaciones fueron: una dehiscencia de la herida (paciente 1), que fue tratada mediante cierre por segunda y curaciones con azúcar; una infección del sitio quirúrgico por Staphylococcus aureus sensible a meticilina, que requirió limpieza quirúrgica y tratamiento antibiótico por seis semanas, sin mayores complicaciones (paciente 3) (Figura 8). Un paciente tuvo una fístula de líquido cefalorraquídeo (caso 2) que se resolvió con drenaje y vendaje compresivo.

Los resultados funcionales obtenidos fueron secundarios y se correlacionaron con el nivel de raíz sacrificada: un caso de disfunción vesical definitiva (paciente 2) que se trata con cateterismo intermitente, ya que hubo que seccionar ambas raíces S2. En el otro caso, se logró preservar solo una raíz S2 (paciente 3) generando una vejiga neurogénica que se mantuvo durante tres meses, con recuperación parcial de la función. El resto de los pacientes tiene funciones vesical e intestinal normales. Ninguno presenta disfunción motora.

Con respecto al seguimiento, no se han detectado recidivas al año de seguimiento (pacientes 1 y 4), a los dos años (paciente 2) y a los seis meses (paciente 3 ).

\section{Discusión}

El tratamiento quirúrgico de los tumores sacros es uno de los grandes desafíos para todo cirujano espinal. La complejidad de la anatomía de la pelvis y la presencia de enfermedad avanzada hace que, muchas veces, se requiera de un abordaje multidisciplinario. Este procedimiento está determinado por numerosos factores, como el estado preoperatorio del paciente, las características anatómicas de la lesión, la zona del sacro comprometida y la biología de tumor en sí. Los pacientes deben ser cuidadosamente seleccionados con respecto a estos puntos, ya que las resecciones sacras son procedimientos de elevada morbi-

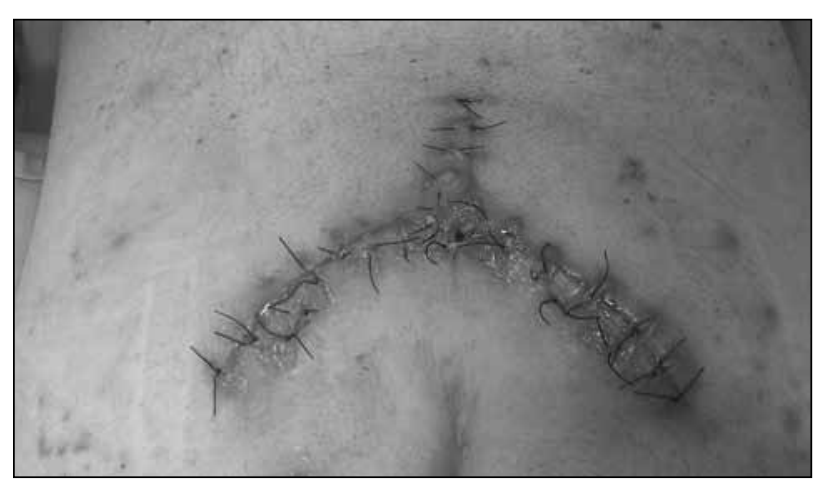

Figura 8. Eritema de los bordes de la herida en un paciente con infección de sitio quirúrgico.

mortalidad, con pérdidas sensitivas y motoras, así como disfunción vesical, intestinal y sexual. ${ }^{7}$

En caso de tumores metastásicos, se han desarrollado varios sistemas de estadificación que ayudan a la correcta selección del paciente. En general, los pacientes con múltiples metástasis vertebrales, en diferentes órganos y pobre estado de salud, no son candidatos para este tipo de cirugía. Los pacientes con patologías tumorales favorables, como las secundarias de mama o próstata, pueden ser buenos candidatos para el tratamiento quirúrgico, aunque presenten metástasis en múltiples sitios. Por otro lado, aquellos con metástasis muy agresivas, como el cáncer de pulmón, no resultan ser buenos candidatos para la cirugía, a pesar de tener lesiones solitarias ${ }^{8-10} \mathrm{En}$ el caso de nuestro paciente, presentaba una lesión metastásica sacra aislada de un carcinoma prostático, sin otros órganos comprometidos, con un muy buen estado general y una expectativa de vida elevada, lo que motivó realizar la cirugía acompañada de un seguimiento urológico por su patología de base.

La resección parcial o total del sacro es considerada el tratamiento de elección para las lesiones malignas primarias, porque permite controlar localmente la lesión y aumentar la tasa de supervivencia. Se debe procurar una resección con márgenes amplios ya que es el factor predictivo más importante para disminuir la recurrencia y aumentar la supervivencia de estos pacientes. ${ }^{11-12}$

Las dificultades terapéuticas de este tipo de patología radican en las grandes masas tumorales que dificultan obtener un buen margen libre de enfermedad y, otras veces, el control local puede alterarse como resultado de la biopsia prequirúrgica. ${ }^{13} \mathrm{~A}$ pesar de que la biopsia guiada por tomografía es un procedimiento seguro, exacto y, en la mayoría de los casos, suficiente para obtener una muestra ósea satisfactoria, creemos que si la imagen es característica, la biopsia prequirúrgica puede no ser necesaria y, de esta forma, disminuye la posibilidad de colonización del trayecto de la aguja. Si existiese duda con respecto al diagnóstico diferencial y se requiere una biopsia percutánea guiada por tomografía se necesita planificar el lugar 
de ingreso y el trayecto para ser resecado en la cirugía. Se han comunicado porcentajes de éxito para llegar a un diagnóstico mediante biopsia guiada por tomografía superiores al $90 \%$ para lesiones líticas y metastásicas sacras. ${ }^{14}$

La literatura provee diferentes sugerencias y experiencias con respecto al mejor abordaje quirúrgico, pero ninguno ha resultado ser el patrón oro debido a la heterogeneidad de los tumores, el tamaño y el compromiso de diferentes órganos pelvianos. La localización del tumor dentro del sacro determina si la resección podrá ser parcial o total con el fin de alcanzar márgenes libres de enfermedad. Las resecciones proximales a S2, muchas veces, requieren abordajes combinados anteriores y posteriores tanto para la resección como para la solución del anillo pelviano, mientras que se prefiere la vía posterior para las resecciones distales..$^{15}$ Cuando la resección sacra no afecte S1, la estabilidad pelviana no se verá comprometida, caso contrario, se deberá realizar una instrumentación lumbopelviana con reconstrucción del anillo pelviano. ${ }^{16}$

En nuestros pacientes, el abordaje lumbosacro por vía posterior en copa invertida hizo posible obtener una excelente exposición del campo operatorio, y se pudo conseguir una resección marginal en los cuatro casos, sin rotura del tumor. La creación de un colgajo que se repliega sobre la línea interglútea nos permite alejarnos de una posible fuente de infección y un mejor control de la herida posoperatoria.

En la mayoría de los pacientes con tumores malignos sacros, la resección pieza a pieza generará una contaminación extensa del campo y un aumento en la tasa de recurrencia superior al 70\%. Esto puede ser fatal, porque las posibilidades de resección marginal en la siguiente cirugía serán nulas. ${ }^{17}$

Como la fascia rectal y el periostio presacro son barreras a la infiltración e invasión tumoral, la pared rectal, rara vez, está involucrada. Sin embargo, los tejidos presacros pueden ser extensos y desplazar las estructuras pelvianas vitales y causar adherencias. La disección digital y la posibilidad de traspasar una gasa vaselinada de lado a lado son de vital importancia para disminuir el riesgo de complicaciones mayores, como lesiones vasculares y rectales.

Los abordajes de este tipo de tumores se asocian a una gran lesión de partes blandas, pérdidas sanguíneas, lesión radicular e infección. Cuando la escisión tumoral es distal a S2, la laminectomía se realiza a partir de L5 y S1, con el fin de visualizar y seguir el trayecto de la raíz $\mathrm{S} 1$ para evitar lesionarla. La preservación de esta raíz conlleva grandes beneficios en lo que respecta a la deambulación posoperatoria. ${ }^{18}$ Inevitablemente este tipo de cirugía trae secuelas neurológicas relacionadas con las funciones intestinal, vesical y sexual, las cuales se sobrellevan satisfactoriamente si se consigue preservar ambas raíces $\mathrm{S} 1$. La preservación de ambas raíces S2 determina una función vesical normal en el $25 \%$ y una función intestinal normal en el $40 \%$ de los casos. Si se logra preservar una raíz S3, estos resultados mejoran a un $60 \%$ para la función vesical y un $67 \%$ para el control intestinal. Si ambas raíces S3 son preservadas la mejoría asciende al $69 \%$ y $100 \%$ para función vesical e intestinal, respectivamente. Si la sección radicular sacra es unilateral, las funciones vesical e intestinal se mantienen normales en más del $80 \% .^{19}$

Según Sciubba y cols., ${ }^{20}$ los riesgos de infección en este tipo de cirugías son mucho más altos que en el resto de las cirugías espinales, probablemente debido a un prolongado tiempo operatorio, al gran daño de partes blandas y a la proximidad del recto dentro del campo operatorio. Se han reportado tasas del $26-46 \%$, algunos de los potenciales factores de riesgo asociados con aumento de infecciones son: diabetes mellitus, tabaquismo, terapia con glucocorticoides, obesidad, niveles de albúmina $<3,5 \mathrm{~g} / \mathrm{dl}$, antecedentes de cirugía y radioterapia preoperatoria, como así también la cantidad de cirujanos que participan en la cirugía, ya que habitualmente son procedimientos multidisciplinarios que involucran cirujanos espinales, plásticos, urólogos, generales. En nuestro caso, el equipo quirúrgico contó con tres cirujanos espinales y una técnica instrumentista en tres casos y, en el restante, participó un neurocirujano. Por todo lo dicho, creemos que es de extrema importancia intentar controlar todos estos factores, en la medida de lo posible, para tratar de disminuir el riesgo de infección.

\section{Conclusiones}

Los tumores sacros son patologías que plantean un desafío diagnóstico y terapéutico. La resección parcial del sacro por abordaje posterior único se puede indicar cuando la lesión compromete desde S2 hacia distal y siempre que no haya compromiso sacroilíaco y puede estar a cargo de cirujanos espinales con experiencia. La preservación de raíces nerviosas es de vital importancia para garantizar mejores resultados posoperatorios y una menor tasa de infección. 


\section{Bibliografía}

1. Sciubba DM, Pettey RJ, Garces Ambrossi GL, Noggle JC, McGirt MJ, Wolinsky JP, et al. Diagnosis and management of sacral tumors. A review. J Neurosurg Spine 2009;10:244-256.

2. Sciubba DM, Chi JH, Rhines LD, Gokaslan ZL. Chordoma of the spinal column. Neurosurg Clin North Am 2008;19:5-15.

3. Kawai A, Healey JH, Boland PJ, Lin PP, Huvos AG, Meyer PA. Prognostic factors for patients with sarcomas of the pelvic bones. Cancer 1998;82:851-859.

4. Park L, Delaney TF, Liebsch NJ, Hormicek FJ, Golberg S, Mankin H, et al. Sacral chordomas: impact of high-dose proton/photon-beam radiation therapy combined with or without surgery for primary versus recurrent tumor. Int J Radiat Oncol Biol Phys 2006;65:1514-1521.

5. McLoughlin GS, Sciubba DM, Wolinsky JP. Chondroma/chondrosarcoma of the spine. Neurosurg Clin North Am 2008;19:57-63.

6. Angelini A, Ruggieri P. New surgical technique of sacral resection. Spine 2013;38(3):E185-E192.

7. Fourney DR, Gokaslan ZL. Surgical approaches for the resection of sacral tumors. En: Dickman CA, Fehlings MG, Gokaslan ZL (eds.). Spinal cord and spinal column tumors: principles and practice, New York: Thieme; 2006:632-648.

8. Tokuhashi Y, Matsuzaki H, Oda H, Oshima M, Ryu J. A revised scoring system for preoperative evaluation of metastatic spine tumor prognosis. Spine 2005;30:2186-2191.

9. Tokuhashi Y, Matsuzaki H, Toriyama S, Kawano H, Ohsaka S. Scoring system for the preoperative evaluation of metastatic spine tumor prognosis. Spine (Phil Pa 1976) 1990;15:1110-1113.

10. Tomita K, Kawahara N, Kobayashi T, Yoshida A, Murakami H, Akamaru T. Surgical strategy for spinal metastases. Spine 2011; 26:298-306.

11. Fourney DR, Gokaslan ZL. Current management of sacral chordoma. Neurosurg Focus 2003;15:E9.

12. Ruggieri P, Angelini A, Ussia G, Montalty M, Mercuri M. Surgical margins and local control in resection of sacral chordomas. Clin Orthop Relat Res 2010;468:2939-2947.

13. Sabuncuoglu H, Ozdogan S, Dogan H, Ataoglu O, Timurkaynak E. Total resection of inferiorly located sacral chordoma. Turkish Neurosurgery 2010;20(4):527-532.

14. Kattapuram SV, Khurana JS, Rosenthal DI. Percutaneous needle biopsy of the spine. Spine 1992;17:561-564.

15. Asavamongkolku A, Waikakul S. Wide resection of sacral chordoma via a posterior approach. Int Orthop 2012;36:607-612.

16. Gallia GL, Haque R, Garoznik I, Witham TF, Khavkin YA, Wolinsky JP, et al. Spinal pelvic reconstruction after total sacrectomy for en bloc resection of a giant sacral chordoma: Technical note. J Neurosurg Spine 2005;3:501-506.

17. Dasen Li, Wei Guo, Xiaodong Tang, Tao Ji, Yidan Zhang. Surgical classification of different types of en bloc resection for primary malignant sacral tumors. Eur Spine J 2011;20:2275-2281.

18. Stener B, Gunterberg B. High amputation of the sacrum for extirpation of tumors: principles and techniques. Spine 1978;3: 351-366.

19. Saigal R, Lu DC. Conversion of high sacral to midsacral amputation via S-2 nerve preservation during partial S-2 sacrectomy for cordoma. J Neurosurg Spine 2014;20:421-429.

20. Sciubba CN. Evaluation of factors associated with postoperative infection following sacral tumor resection. J Neurosurg Spine 2008;9:593-599. 